\title{
Armut in der Stadt - Prozesse der Ausweitung und Verfestigung räumlich segregierter Armut am Beispiel der Stadt Bielefeld
}

\section{Andreas Farwick, Bremen}

Seit Beginn der 1980er Jahre hat sich das Ausmaß der Armut in der Bundesrepublik Deutschland zunehmend verschärft und ist zu einem zentralen sozialpolitischen Problem geworden. Insbesondere in den Städten und hier wiederum in bestimmten städtischen Teilgebieten steigt die Zahl der von Armut betroffenen Bevölkerung erheblich an (z. B. KLAGGE 1998). Untersuchungen in verschiedenen westdeutschen Städten zeigen, daß sich die Armutsbevölkerung gerade in citynahen Stadtgebieten mit ungünstigen Wohnbedingungen und in den randstädtischen Großwohnanlagen des öffentlich geförderten sozialen Wohnungsbaus der 1960er und 1970er Jahre konzentriert (vgl. u. a. ReICHERTZ 1987; LandeshaupTSTADT StUTTGART 1989; FreIE und Hansestadt Hamburg 1993; Farwick 1998).

Während die Vor- und Nachteile einer sozialräumlichen Segregation benachteiligter Bevölkerungsgruppen in den 1960er und 1970er Jahren unter dem Schlagwort «Homogenität vs. Heterogenität von Wohnquartieren» (HerlyN 1974; BAHRDT 1974; VASKovics 1982) kontrovers diskutiert wurden, überwiegen mit dem Anstieg räumlich konzentrierter Armut und der damit verbundenen Kumulation sozialer Probleme in immer stärkerem Maße negative Beurteilungen (z. B. Froessler 1994, Kronauer 1997, HäUsSermanN \& SieBeL 2000). Beschreibungen der Lebenssituation von Armut Betroffener in den Armutsgebieten der Großstädte zeichnen ein Bild, das bisher nur aus Darstellungen über die Verhältnisse in den Obdachlosensiedlungen der 1970er Jahre (vgl. Hess \& MECHLER 1972, VASKOVICS 1976) bekannt war. So wird berichtet, daß die Stigmatisierung und Diskriminierung der Bevölkerung aufgrund der hohen Armutsdichte in diesen Gebieten zunehmen. Im Zuge einer erlebten sozialen und auch räumlichen Ausgrenzung komme es zu Rückzugsverhalten, Apathie und Resignation (Naroska 1987, Freie UND HaNSESTADT HaMBURG 1993). Untersuchungen von FARWICK (FARWICK 1998, 2001) können den benachteiligenden Einfluß räumlich segregierter Armut im Hinblick auf die Dauer von Armutslagen bestätigen. So steigt in Wohnquartieren mit hoher Sozialhilfedichte das Risiko eines langfristigen Sozialhilfebezugs deutlich an.

Vor dem Hintergrund der mit der räumlichen Konzentration sozial benachteiligter Bevölkerungsgruppen verbunden Problematik erscheint es als verwunderlich, daß bisher wenig über die Prozesse und Mechanismen eines räumlich disparitär verlaufenden Anstiegs der Armut in den Städten bekannt ist. So ist bisher ungeklärt, in welchem Ausmaß die räumliche Segregation von Armut auf eine Verarmung der Bevölkerung in bestimmten Stadtgebieten zurückzuführen ist, oder/ und ob diese durch eine Zuwanderung bereits von Armut Betroffener oder/und durch eine Abwanderung der nicht von Armut betroffenen Bevölkerung hervorgerufen wird. Soll eine sozialpolitische Intervention im Sinne einer Stadtentwicklungsplanung des sozialen und räumlichen Ausgleichs erfolgreich betrieben werden, so sind Kenntnisse über die Prozesse einer räumlichen Segregation jedoch unabdingbar.

In diesem Beitrag wird zunächst das Ausmaß und die räumliche Verteilung besonders stark von Armut betroffener Gebiete in der Stadt Bielefeld untersucht. Aufgrund der Verfügbarkeit von kleinräumigen amtlichen Statistiken und prozeßproduzierter Individualdaten wird Armut in den vorliegenden Analysen durch den Bezug von Sozialhilfe - genauer Hilfe zum Lebensunterhalt (HLU) - definiert.

Den Schwerpunkt des Beitrags bildet die Analyse von Individualdaten der Bielefelder Datenbank «Sozialhilfestatistik». Diese Daten erlauben es, die räumliche Verteilung und Mobilität von Sozialhilfeempfängern nachzuverfolgen und so neue Erkenntnisse über Prozesse der Segregation und über die räumliche Ungleichverteilung von Armut in der Stadt zu gewinnen.

\section{Die Entwicklung segregierter Armut in Bielefeld}

Bevor im folgenden auf die räumliche Konzentration der Wohnstandorte von Sozialhilfeempfängern eingegangen wird, soll zunächst die zeitliche Entwicklung von Armut in der Stadt Bielefeld aufgezeigt werden.

Da die Arbeitslosigkeit seit Mitte der 1970er Jahre eine wesentliche Ursache für Armut und Sozialhilfebezug darstellt, ist die Entwicklung der Zahl der Sozialhilfeempfänger eng mit der Dynamik auf dem Arbeitsmarkt verbunden. Arbeitslosigkeit führt zumeist erst nach einer längeren Zeitphase - häufig nach Ausschöpfung des Anspruchs auf Arbeitslosengeld - zum Bezug von Sozialhilfe. Aus diesem Grund folgt der Verlauf der Sozialhilfedichte, definiert als 


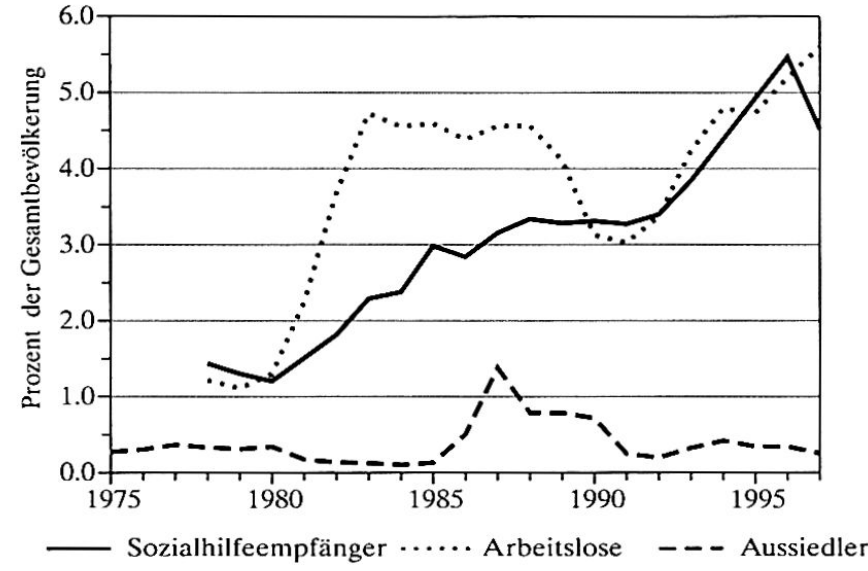

Abb. 1: Entwicklung der Zahl der Sozialhilfeempfänger und der Arbeitslosen am Jahresende sowie der Aussiedlerzugänge in Prozent der Gesamtbevölkerung

Development of the number of social welfare recipients and unemployed at the end of a year as well as the increase of German immigrants in percent of the total population

Evolution du nombre des bénéficiaires de l'aide sociale, des personnes sans emploi à la fin de l'année et des émigrants en pour-cent de la population totale Quelle: Stadt Bielefeld, Amt für Stadtforschung und Statistik, Sozialamt; Arbeitsamt Bielefeld prozentualer Anteil der Sozialhilfeempfänger an der Bevölkerung, dem Ausmaß der Arbeitslosigkeit weniger dynamisch und zeitlich etwas versetzt.

Abbildung 1 zeigt, daß die Sozialhilfedichte in Bielefeld seit Anfang der 1980er Jahre beständig angestiegen ist. Ein Hauptgrund für die zunehmende Armut ist die hohe Arbeitslosigkeit seit der Rezessionsphase zu Beginn der 1980er Jahre. Mit der erwähnten zeitlichen Verzögerung zwischen dem Beginn von Arbeitslosigkeit und Sozialhilfebezug und dem sprunghaften Anstieg der Zahl der Aussiedler (die in der Regel nach Ankunft in der Bundesrepublik durch Sozialhilfeleistungen unterstützt werden) seit 1985 ist es zu erklären, daß die Sozialhilfedichte bis 1988 weiter anstieg, obwohl die Zahl der Arbeitslosen ab 1983 stagnierte. Während die Arbeitslosigkeit im Zuge des kurzen wirtschaftlichen Aufschwungs nach der Wiedervereinigung bis 1991 deutlich zurückging, blieb die Zahl der Sozialhilfeempfänger aufgrund des Zuzugs von Aussiedlern und Asylbewerbern (die bis zum Inkrafttreten des Asylbewerberleistungsgesetzes im Jahre 1994 ebenfalls durch Sozialhilfe unterstützt wurden) konstant und stieg bis 1996 auf einen Höchstwert von 5,5 Prozent an, bevor sie im Jahr 1997 auf 4,5 Prozent absank.

Wird nun die räumliche Verteilung der Sozialhilfeempfänger in Bielefeld betrachtet, so verweist Karte 1

\begin{tabular}{|lccccccc|}
\hline & \multicolumn{2}{c}{ Bevölkerung } & \multicolumn{2}{c}{$\begin{array}{c}\text { Sozialhilfe- } \\
\text { empfänger }\end{array}$} & \multicolumn{3}{c|}{ Sozialhilfedichte } \\
& $\mathbf{1 9 9 2}$ & $\mathbf{1 9 9 6}$ & $\mathbf{1 9 9 2}$ & $\mathbf{1 9 9 6}$ & $\mathbf{1 9 9 2}$ & $\mathbf{1 9 9 6}$ & Differenz \\
\hline Bauernschaft & & & & & & & \\
Schildesche & 1964 & 1940 & 92 & 273 & 4,7 & 14,1 & $+9,4$ \\
Kesselbrink & 2827 & 2597 & 210 & 279 & 7,4 & 10,7 & $+3,3$ \\
Dürkopp & 2023 & 2023 & 187 & 202 & 9,2 & 10,0 & $+0,8$ \\
Baumheide & 8104 & 8251 & 364 & 787 & 4,5 & 9,5 & $+5,0$ \\
Sieker & 4510 & 4431 & 208 & 411 & 4,6 & 9,3 & $+4,7$ \\
Pauluskirche & 3429 & 4157 & 200 & 374 & 5,8 & 9,0 & $+3,2$ \\
Stadtwerke & 2726 & 2726 & 124 & 201 & 4,6 & 7,4 & $+2,8$ \\
Osningpaß & 1102 & 1017 & 26 & 73 & 2,4 & 7,2 & $+4,8$ \\
Fuhrpark & 2526 & 2277 & 137 & 159 & 5,4 & 7,0 & $+1,6$ \\
Milse & 5241 & 6068 & 121 & 422 & 2,3 & 7,0 & $+4,7$ \\
Schildesche & 2505 & 2305 & 138 & 159 & 5,5 & 6,9 & $+1,4$ \\
Oldentrup-West & 3755 & 4057 & 128 & 279 & 3,4 & 6,9 & $+3,5$ \\
\hline Bielefeld insg. & 320459 & 321366 & 8144 & 14042 & 2,5 & 4,4 & $+1,9$ \\
\hline
\end{tabular}

Sozialhilfedichte $=$ prozentualer Anteil der Sozialhilfeempfänger an der Bevölkerung

Armutsgebiet im jeweiligen Jahr

überdurchschnittlicher Anstieg der Sozialhilfedichte

Tab. 1: Die Armutsgebiete der Stadt Bielefeld 1992 und 1996 (Angaben jeweils am Jahresende)

Poverty areas in the city of Bielefeld, 1992 and 1996 (year-end figures)

Les zones de pauvreté dans la ville de Bielefeld en 1992 et 1996 (données de la fin de chaque année)

Quelle: Stadt Bielefeld, Amt für Stadtforschung und Statistik; Bielefelder Datenbank «Sozialhilfestatistik» (Sozialamt Bielefeld; Universität Bielefeld) 


\section{Sozialhilfedichte}

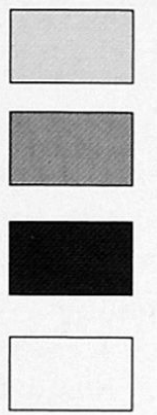

unterdurchschnittlich (unter 4,0\%)

überdurchschnittlich $(4,0 \%$ bis unter $6,7 \%)$

Gebiete hoher Sozialhilfedichte $(6,7 \%$ und höher $)$

Gebiete mit geringer Bevölkerungsdichte

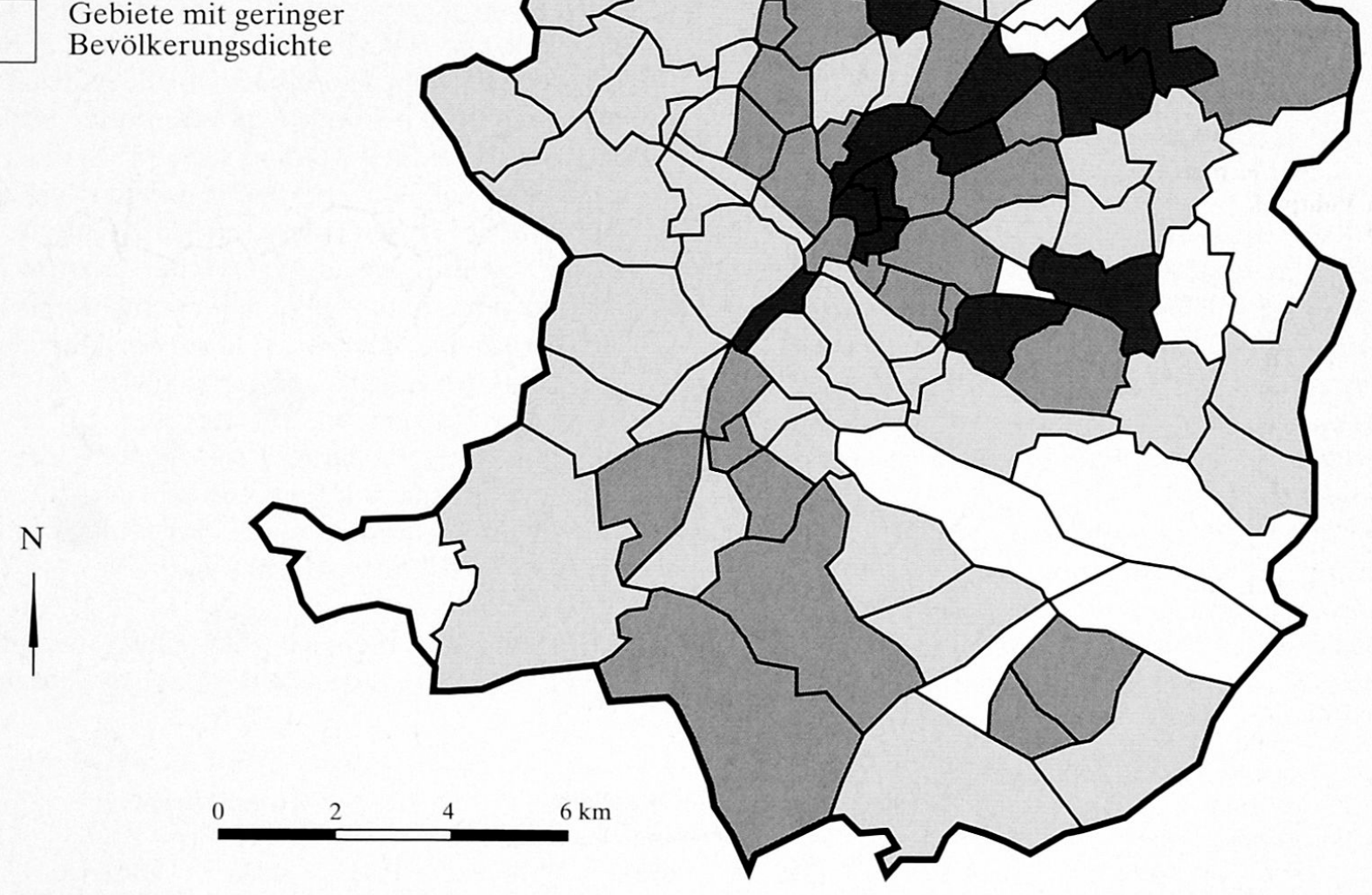

Karte 1: Dichte der Sozialhilfeempfänger (HLU) in den Bielefelder Statistischen Bezirken am Jahresende 1996 Density of social welfare recipients in the Bielefeld census districts, December 1996

Densité des bénéficiaires de l'aide sociale dans les districts statistiques de la ville de Bielefeld, fin 1996

Quelle: Stadt Bielefeld, Amt Für StadtForschung und Statistik; Bielefelder Datenbank «Sozialhilfestatistik» (Sozialamt Bielefeld; Universität Bielefeld); Kartengrundlage: Amt für Stadtforschung und Statistik; Bearbeitung: A. FARWICK

auf eine deutliche Ungleichverteilung von Armut im Bielefelder Stadtgebiet. Insbesondere zwölf Statistische Bezirke sind in hohem Maße von Armut betroffen. In diesen Gebieten übersteigt die Sozialhilfedichte den ungewichteten Mittelwert der Sozialhilfedichten aller Statistischer Bezirke um mehr als eine Standardabweichung. Gebiete mit einer-gemessen am städtischen Durchschnitt - derartig hohen Armutsausprägung werden im folgenden als Armutsgebiete bezeichnet. Im wesentlichen handelt es sich bei diesen Armutsgebieten um unattraktive citynahe Gebiete, traditionelle Arbeitergebiete und Gebiete mit Großwohnanlagen des sozialen Wohnungsbaus. Ein
Armutsgebiet (Milse) ist durch eine randstädtische Lage mit aufgelockerter Siedlungsstruktur gekennzeichnet. Dieser Stadtteil zeichnet sich durch eine Neubebauung mit Übergangswohnhäusern für Aussiedler aus.

Die Entwicklung der Sozialhilfedichte in den Armutsgebieten für den Zeitraum von 1992 bis 1996 (vgl. Tabelle 1) ist in drei der zwölf Armutsgebiete von 1996 (Dürkopp, Fuhrpark und Schildesche) durch einen -im Vergleich zur Gesamtstadt - unterdurchschnittlichen Anstieg auf bereits hohem Niveau geprägt. Gemessen an der Situation in der Gesamtstadt ist in diesen Gebie- 
1 Alt- und Neustadt

2 Kesselbrink

3 Pauluskirche

4 Dürkopp

5 Landgericht

6 Upmannstift

7 Siegfriedplatz

8 Nordpark

9 Stadtwerke

10 Güterbahn-Ost

11 Hammer-Mühle

12 Königsbrügge

13 Brands Busch

14 Betriebshof Sieker

15 Grossmarkt

16 Stauteiche

17 Heeper Fichten

18 Fuhrpark

19 Kammerratsheide

20 Bauernschaft Schildesche

21 Vorwerk Schildesche

22 Schildesche

23 Johannesstift

24 Sudbrack

25 Untertheesen

26 Bültmannskrug

27 Gellershagen

28 Bültmannshof

29 Universität

30 Sieben Hügel

31 Johannistal

32 Osningpass

33 Bethel

34 Eggeweg

35 Rosenhöhe

36 Kammerich

37 Frerks Hof

38 Brackwede-Mitte

39 Bahnhof Brackwede

40 Kupferhammer

41 Brock

42 Südwestfeld

43 Ummeln

44 Holtkamp

45 Kupferheide

46 Quelle

47 Kirchdornberg

48 Hoberge-Ürentrup

49 Wolfskuhle

50 Wellensiek

51 Pappelkrug

52 Grossdornberg

53 Babenhausen-Ost

54 Babenhausen

55 Niederdornberg-Schröttinghausen

56 Jöllenbeck-West

57 Jöllenbeck-Ost

58 Theesen

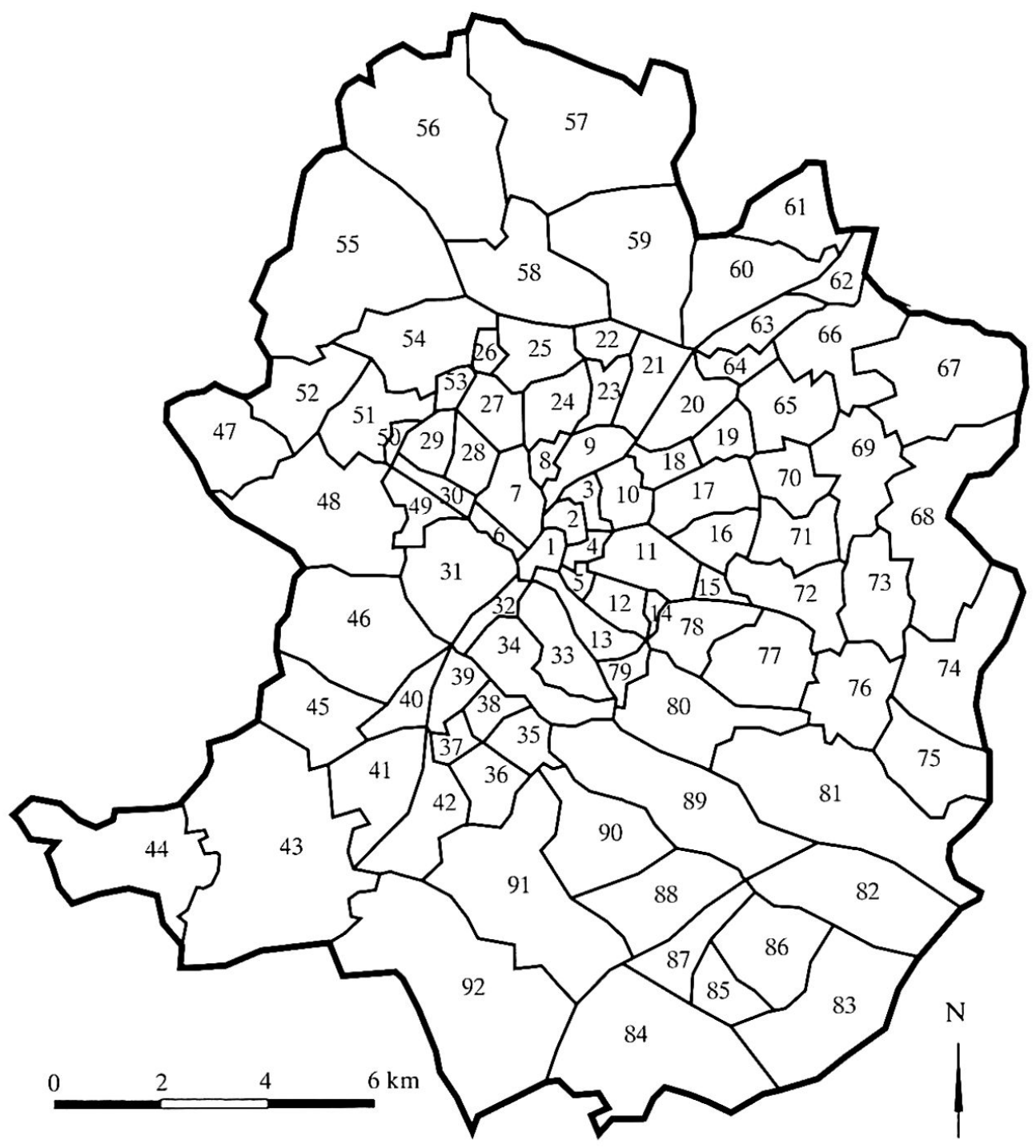

59 Vilsendorf

60 Grafenheide

61 Lämmkenstatt

62 Welscher

63 Jerrendorf

64 Halhof

65 Baumheide

66 Milse

67 Altenhagen

68 Brönninghausen

69 Windwehe

70 Tieplatz

71 Heeper Holz

72 Oldentrup-West

73 Oldentrup-ost

74 Dingerdissen

75 Ubbedissen

76 Hillegossen
77 Stieghorst

78 Sieker

79 Lonnerbach

80 Rütli

81 Lämershagen

82 Wrachtruper Lohde

83 Dalbke

84 Eckardtsheim

85 Südstadt

86 Sennestadt

87 Sennestadt-Industriegebiet

88 Schillingshof

89 Todrang

90 Buschkamp

91 Windelsbleiche

92 Windflöte

Karte 2: Stadt Bielefeld, Statistische Bezirke

City of Bielefeld, statistical districts

La ville de Bielefeld, les districts statistiques

Kartengrundlage: Amt FÜr STADTFORSCHUNG Und Statistik; Bearbeitung: A. Farwick 


\begin{tabular}{|c|c|c|c|c|c|c|c|c|c|c|c|}
\hline $\begin{array}{l}\text { Statistischer } \\
\text { Bezirk }\end{array}$ & $\begin{array}{c}\text { Soz. } \\
92 \\
\left(a_{10}\right)\end{array}$ & $\begin{array}{c}\text { Bev. } \\
92 \\
\left(b_{10}\right)\end{array}$ & $\begin{array}{c}\text { Soz.-D. } \\
92 \\
\left(d_{10}\right)\end{array}$ & $\begin{array}{c}\text { Soz. } \\
96 \\
\left(a_{11}\right)\end{array}$ & $\begin{array}{c}\text { Bev. } \\
96 \\
\left(b_{11}\right)\end{array}$ & $\begin{array}{c}\text { Soz.-D. } \\
96 \\
\left(d_{11}\right)\end{array}$ & $\begin{array}{c}\text { Soz. } \\
96 \text { bei } \\
\text { Bev. } 96 \\
\left(a_{b t 1}\right) \\
\end{array}$ & $\begin{array}{c}\text { Soz. } \\
96 \text { bei } \\
\text { Bev.92 } \\
\left(\mathrm{a}_{\mathrm{bt0}}\right) \\
\end{array}$ & $\begin{array}{c}a_{t 1}-a_{b t 1} \\
\text { (A) }\end{array}$ & $\begin{array}{c}a_{b t 0}-a_{b t 1} \\
\text { (B) }\end{array}$ & $\begin{array}{c}\mathbf{a}_{\mathrm{ti}}-\mathrm{a}_{\mathrm{bt0}} \\
\text { (C) }\end{array}$ \\
\hline Bauernschaft & & & & & & & & & & & \\
\hline Schildesche & 92 & 1964 & 4,7 & 273 & 1940 & 14,1 & 126 & 128 & 147 & 2 & 145 \\
\hline Kesselbrink & 210 & 2827 & 7,4 & 279 & 2597 & 10,7 & 240 & 262 & 39 & 21 & 17 \\
\hline Baumheide & 364 & 8104 & 4,5 & 787 & 8251 & 9,5 & 521 & 512 & 266 & -9 & 275 \\
\hline Sieker & 208 & 4510 & 4,6 & 411 & 4431 & 9,3 & 285 & 290 & 126 & 5 & 121 \\
\hline Pauluskirche & 200 & 3429 & 5,8 & 374 & 4157 & 9,0 & 318 & 263 & 56 & -56 & 111 \\
\hline Stadtwerke & 124 & 2726 & 4,5 & 201 & 2726 & 7,4 & 174 & 174 & 27 & 0 & 27 \\
\hline Osningpaß & 26 & 1102 & 2,4 & 73 & 1017 & 7,2 & 43 & 46 & 30 & 4 & 27 \\
\hline Milse & 121 & 5241 & 2,3 & 422 & 6068 & 7,0 & 251 & 217 & 171 & -34 & 205 \\
\hline Oldentrup-West & 128 & 3755 & 3,4 & 279 & 4057 & 6,9 & 212 & 197 & 67 & -16 & 82 \\
\hline Armutsgebiete & 1473 & 33658 & 4,4 & 3099 & 35244 & 8,8 & 2156 & 2088 & 943 & -68 & 1011 \\
\hline übrige Stadt & 6671 & 286801 & 2,3 & 10943 & 286122 & 3,8 & 11886 & 11914 & -943 & 28 & -971 \\
\hline Bielefeld insg. & 8144 & 320459 & 2,5 & 14042 & 321366 & 4,4 & 14042 & 14002 & 0 & -40 & 40 \\
\hline
\end{tabular}

$a_{b t 1}=a_{t 0}+\left(b_{t 1}-b_{t 0}\right)\left(d_{10} / 100\right)+b_{t 1}\left(\left(D_{11}-D_{t 0}\right) / 100\right)$ unter der Prämisse eines gleichen Zuwachses der Sozialhilfedichte (in Prozentpunkten) wie in der Gesamtstadt, bei Bev. wie zum Zeitpunkt $t_{1}$

$a_{b t 0}=a_{t 0}+b_{t 0}\left(\left(D_{t 1}-D_{t 0}\right) / 100\right)$ unter der Prämisses eines gleichen Zuwachses der Sozialhilfedichte (in Prozentpunkten) wie in der Gesamtstadt, bei Bev. wie zum Zeitpunkt $t_{0}$

Bedeutung der Abkürzungen: Soz. = Sozialhilfeempfänger, Bev. = Bevölkerung, Soz.-D. = Sozialhilfedichte, $\mathrm{D}_{1}=$ Sozialhilfedichte der Gesamtstadt zu einem jeweiligen Zeitpunkt. Bevölkerungszahlen am Jahresende.

Tab. 2: Zerlegung des Gesamteffekts eines überproportionalen Anstiegs der Sozialhilfedichte in den Armutsgebieten (1992 bis 1996)

Closer analysis of an overall increase in the poverty rate in poverty areas (1992 to 1996)

Analyse de l'effet global d'un accroissement exagéré de la densité de l'aide sociale dans les zones de pauvreté (de 1992 à 1996) Quelle: Stadt Bielefeld, Amt Für StadTforschung und Statistik; Bielefelder Datenbank «Sozialhilfestatistik» (Sozialamt Bielefeld; Universität Bielefeld)

ten also eine leichte Entspannung der räumlichen Konzentration von Armut zu verzeichnen. In neun der zwölf Armutsgebiete des Jahres 1996 hat demgegenüber ein im Vergleich zur Gesamtstadt überdurchschnittlicher Anstieg der Sozialhilfedichte stattgefunden. Aufgrund dieser überdurchschnittlichen Zunahme der Sozialhilfedichte in bestimmten Teilgebieten der Stadt haben sich einerseits neue Gebiete hoher Sozialhilfedichte gebildet (Osningpaß, Milse und Oldentrup-West), andererseits fand eine Verfestigung von Armut in bereits bestehenden Armutsgebieten statt.

Die überdurchschnittliche Zunahme der Sozialhilfedichte in der Mehrzahl der Armutsgebiete in Bielefeld deutet auf Tendenzen der sozialräumlichen Polarisierung hin, wie sie seit längerem unter dem Begriff der «dual city» (vgl. Mollenkopf \& CASTElls 1991) oder der «dreigeteilten Stadt» (vgl. HäUSSERMANN \& Siebel 1987) debattiert werden.

\section{Ursachen der räumlichen Konzentration von Sozialhilfeempfängern}

Werden die Ursachen der überdurchschnittlichen Zunahme der Sozialhilfedichte in der Mehrzahl der
Armutsgebiete weiter verfolgt, so ist zunächst festzuhalten, daß die Befunde bisher noch keinen Rückschluß auf die Entwicklung der Armut in den betroffenen Armutsgebieten zulassen, da eine Veränderung der Sozialhilfedichte nicht nur durch die Entwicklung der Armutsbevölkerung im jeweiligen Teilgebiet, sondern auch durch die Entwicklung der Gesamtbevölkerung im Teilgebiet (ohne jede Veränderung der Zahl der Sozialhilfeempfänger) beeinflußt sein kann. So würde z. B. ein massiver Fortzug der «wohlhabenden» Bevölkerung bei einer konstanten Armutsbevölkerung einen deutlichen Anstieg der Sozialhilfedichte zur Folge haben.

Um nun beurteilen zu können, inwieweit der überdurchschnittliche Anstieg der Sozialhilfedichte in den Armutsgebieten auf eine Zunahme der Armutsbevölkerung in diesen Gebieten zurückzuführen ist (und nicht allein auf Veränderungen der Gesamtbevölkerung beruht), wird der Effekt des überproportionalen Anstiegs der Sozialhilfedichte (A) ähnlich wie bei einer Shift-Analyse (zum Verfahren der Shift-Analyse vgl. z. B. SchäTzL 1981: 46ff) in zwei Teileffekte (B) und (C) zerlegt (vgl. Tabelle 2). Beide Teileffekte summieren sich zu dem Gesamteffekt des überproportionalen Anstiegs bzw. Rückgangs 
der Sozialhilfedichte auf. Damit gilt $\mathrm{A}=\mathrm{B}+\mathrm{C}$, wobei die einzelnen Effekte im folgenden erläutert werden:

(A) beschreibt die Differenz zwischen der tatsächlichen Zahl von Sozialhilfeempfängern zum Zeitpunkt $\mathrm{t}_{1}$ und einer zu erwartenden Zahl von Sozialhilfeempfängern zum Zeitpunk $\mathrm{t}_{1}$, die sich ergäbe, wenn sich die Sozialhilfedichte im Teilgebiet entsprechend der Gesamtstadt und die Bevölkerung im Teilgebiet real entwikkeln würde. Ein positiver Wert der Differenz bedeutet damit eine überproportionale Zunahme, ein negativer Wert eine unterproportionale Zunahme oder sogar Abnahme der Sozialhilfedichte (Gesamteffekt).

(B) beschreibt die Differenz zwischen einer zu erwartenden Zahl von Sozialhilfeempfängern zum Zeitpunkt $t_{1}$, die sich ergäbe, wenn sich die Sozialhilfedichte im Teilgebiet entsprechend der Gesamtstadt entwickelte und außerdem die Bevölkerung im Teilgebiet konstant bliebe und einer zu erwartenden Zahl von Sozialhilfeempfängern zum Zeitpunkt $t_{1}$, die sich ergäbe, wenn sich die Sozialhilfedichte im Teilgebiet entsprechend der Gesamtstadt und die Bevölkerung im Teilgebiet real entwickeln würde. Die Variable B erklärt damit den Teil der Variable A, der ausschließlich auf eine Veränderung der Gesamtbevölkerung im Teilgebiet zurückzuführen ist. Ein positiver Wert der Differenz bedeutet damit eine überproportionale Zunahme, ein negativer Wert eine unterproportionale Zunahme oder sogar Abnahme der Sozialhilfedichte (Effekt einer Veränderung der Gesamtbevölkerung im Teilgebiet).

(C) beschreibt die Differenz zwischen der tatsächlichen Zahl von Sozialhilfeempfängern zum Zeitpunkt $\mathrm{t}$, und einer zu erwartenden Zahl von Sozialhilfeempfängern zum Zeitpunkt $t_{1}$, die sich ergäbe, wenn sich die Sozialhilfedichte im Teilgebiet entsprechend der Gesamtstadt entwickeln würde und die Bevölkerung im Teilgebiet konstant bliebe. Die Variable C erklärt damit den Teil der Variable A, der ausschließlich auf Veränderungen der Zahl der Sozialhilfeempfänger des Teilgebiets zurückzuführen ist. Ein positiver Wert der Differenz bedeutet damit eine überproportionale Zunahme, ein negativer Wert eine unterproportionale Zunahme oder sogar Abnahme der Sozialhilfedichte (Effekt einer Veränderung der Sozialhilfeempfänger im Teilgebiet).

Die Werte der letzten drei Spalten in Tabelle 2 zeigen, daß der in neun von zwölf Armutsgebieten zu verzeichnende überdurchschnittliche Anstieg der Sozialhilfedichte (Gesamteffekt A) in acht Fällen hauptsächlich durch eine überproportionale Zunahme der Zahl der Sozialhilfeempfänger (stark positiver Effekt C) und nur zu einem geringen Teil auf eine Abnahme der Gesamtbevölkerung in den Armutsge- bieten (Effekt B) begründet ist. In nur einem Statistischen Bezirk (Kesselbrink) ist die überdurchschnittliche Zunahme der Sozialhilfedichte größtenteils durch die Abnahme der Gesamtbevölkerung im Teilgebiet verursacht.

Der überproportionale Anstieg der Sozialhilfeempfänger in den acht Armutsgebieten kann wiederum auf folgende Weise verursacht sein: durch

a) den Zuzug bereits von Armut betroffener Bevölkerungsgruppen in die Armutsgebiete;

b) eine im Vergleich zur Gesamtstadt überproportionale Verarmung der ansässigen Bevölkerung in diesen Gebieten;

c) eine im Vergleich zur Gesamtstadt überproportionale Zuweisung von Aussiedlern mit anschließendem Sozialhilfebezug in diese Gebiete;

d) eine Kumulation der Armutsbevölkerung aufgrund eines überdurchschnittlich hohen Anteils dauerhaft von Armut betroffener Bevölkerungsgruppen.

Diese vier Hypothesen schließen sich gegenseitig nicht aus, sondern können sich im Gegenteil überlagern. Ihr Erklärungsgehalt bezieht sich entweder auf Prozesse einer direkten (auch kommunal gesteuerten) Segregation von Sozialhilfeempfängern und Aussiedlern in die Armutsgebiete oder sie setzen bereits bestehende räumliche Konzentrationen von Bevölkerungsgruppen mit erhöhtem Armutsrisiko bzw. Merkmalen langfristiger Armut in den Armutsgebieten voraus.

Derartige Segregationsmuster sind aufgrund eines seit Beginn der 1980er Jahre bestehenden Nachfrageüberhangs auf dem Vermietungsmarkt preisgünstiger Wohnungen nur zu plausibel. Armutsnahe oder bereits verarmte Bevölkerungsgruppen sind aufgrund der Mangelsituation an preisgünstigem Wohnraum verstärkt auf den unattraktiven und daher schwer vermittelbaren innenstädtischen Restwohnbestand, vor allem aber auf die Sozialwohnungen randstädtischer Großwohnanlagen verwiesen (vgl. KreIBICH 1990).

$\mathrm{Zu}$ erklären ist der Nachfrageüberhang im Teilsegment preisgünstiger Wohnungen zum einen durch den enormen Anstieg der Zahl von Arbeitslosen und Sozialhilfeempfängern (vgl. Abbildung 1). Diese Entwicklung führte zwangsläufig zu einer Steigerung der Nachfrage auf diesem Teilwohnungsmarkt. Zum anderen ist die prekäre Wohnungsmarktlage einkommensarmer Bevölkerungsgruppen durch einen starken Rückgang des Bestands preisgünstiger, zumeist innerstädtischer Altbauwohnungen und Sozialwohnungen aufgrund zweier seit längerem anhaltender Entwicklungen auf dem Wohnungsmarkt verursacht.

Zunächst sei hier die seit dem Ende der 1970er Jahre zu beobachtende Aufwertung preisgünstigen innenstadt- 
nahen Wohnraums (Gentrification) genannt. Aufgrund veränderter Haushaltsstrukturen und Lebensstile hat die Attraktivität innenstadtnaher Standorte für einkommensstärkere Haushalte deutlich zugenommen. Durch Umwandlungen von Miet- in Eigentumswohnungen und Mietpreissteigerungen infolge von Modernisierungsmaßnahmen ist ein erheblicher Teil des preisgünstigen innerstädtischen Wohnungsbestands einkommensschwachen Gruppen nicht mehr zugänglich (vgl. Blasius \& Dangschat 1990, Friedrichs \& KeCSKES 1996).

Beschleunigt wird das Schrumpfen des Teilmarktes preisgünstiger Wohnungen zusätzlich durch den seit den 1980er Jahren zu beobachtenden Rückzug des Staates aus dem sozialen Wohnungsbau (vgl. HäUSSERMANN \& SiEbEl 1996) und insbesondere durch die hohe Zahl auslaufender Mietpreis- und Belegungsbindungen im Bestand preisgünstiger innerstädtischer Sozialwohnungen. Die nach dem Krieg einsetzende staatliche Bezuschussung des privaten Mietwohnungsbaus mit der Auflage der Mietpreisbindung hatte lange Zeit die Durchmischung der Einkommensschichten innerstädtischer Wohngebiete gesichert.

Die Reduktion des Bestands an Sozialwohnungen einerseits und der zunehmende Nachfragedruck auf diesen Bestand andererseits macht es den Wohnungsämtern seit langem immer schwerer, eine sozial ver- trägliche Belegungspolitik zu betreiben. So sind die Kommunen in immer stärkerem Maße gezwungen, die Vermittlung von Sozialwohnungen auf Zielgruppen zu konzentrieren, die keine oder nur eingeschränkte Chancen haben, sich auf dem freien Wohnungsmarkt zu versorgen. Dabei wird die räumliche Konzentration wohnungspolitischer Problemgruppen in den unattraktiven und durch hohe Fortzugsraten einkommensstärkerer Haushalte gekennzeichneten Großwohnanlagen zwangsläufig in Kauf genommen (KREIBICH 1985, NAROSKA 1987).

\section{Analyse von Prozessen der Ausweitung und Verfestigung räumlich segregierter Armut}

Die Frage, welche der oben genannten Prozesse den überdurchschnittlichen Anstieg der Sozialhilfeempfänger begründen, läßt sich mit Daten der Bielefelder Datenbank «Sozialhilfestatistik» überprüfen. In dieser an der Universität Bielefeld geführten Datenbank sind Sozialhilfeabrechnungsdaten aufbereitet, die im Rahmen der computergestützten Fallbearbeitung bei der Stadt Bielefeld generiert worden sind (vgl. Andress \& Kemper 1992). Die Datenbank umfaßt Bestandsdaten weitgehend aller Empfänger von Sozialhilfe im Stadtgebiet Bielefeld bis zum Jahre 1996. In einer sogenannten Sozialhilfeempfängerdatei (SEDA) stehen jeweils am Jahresende erhobene Querschnittsin-

\begin{tabular}{|c|c|c|c|c|c|c|c|c|}
\hline \multirow[t]{2}{*}{ Gebietstyp } & \multicolumn{2}{|c|}{$\begin{array}{c}\text { Kohorte } \\
1992\end{array}$} & \multicolumn{2}{|c|}{$\begin{array}{c}\text { Kohorte } \\
1993\end{array}$} & \multicolumn{2}{|c|}{$\begin{array}{c}\text { Kohorte } \\
1994\end{array}$} & \multicolumn{2}{|c|}{$\begin{array}{c}\text { Kohorte } \\
1995\end{array}$} \\
\hline & $a$ & b & $\mathrm{a}$ & $\mathrm{b}$ & $\mathrm{a}$ & b & $\mathrm{a}$ & $\mathrm{b}$ \\
\hline $\begin{array}{l}\text { übrige Stadt } \\
\text { (n) }\end{array}$ & $\begin{array}{l}88 \% \\
(226)\end{array}$ & $\begin{array}{l}87 \% \\
(222)\end{array}$ & $\begin{array}{l}83 \% \\
(294)\end{array}$ & $\begin{array}{l}81 \% \\
(287)\end{array}$ & $\begin{array}{l}84 \% \\
(517)\end{array}$ & $\begin{array}{l}83 \% \\
(510)\end{array}$ & $\begin{array}{l}83 \% \\
(816)\end{array}$ & $\begin{array}{l}83 \% \\
(820)\end{array}$ \\
\hline $\begin{array}{l}\text { Armutsgebiete mit über- } \\
\text { durchschn. Verarmung (n) }\end{array}$ & $\begin{array}{l}12 \% \\
(30)\end{array}$ & $\begin{array}{l}13 \% \\
(34)\end{array}$ & $\begin{array}{l}17 \% \\
(59)\end{array}$ & $\begin{array}{l}19 \% \\
(66)\end{array}$ & $\begin{array}{l}16 \% \\
(99)\end{array}$ & $\begin{array}{l}17 \% \\
(106)\end{array}$ & $\begin{array}{l}17 \% \\
(169)\end{array}$ & $\begin{array}{l}17 \% \\
(165)\end{array}$ \\
\hline gesamt (n) & \multicolumn{2}{|c|}{256} & \multicolumn{2}{|c|}{353} & \multicolumn{2}{|c|}{616} & \multicolumn{2}{|c|}{985} \\
\hline Signifikanz & \multicolumn{2}{|c|}{0,437} & \multicolumn{2}{|c|}{0,318} & \multicolumn{2}{|c|}{0,443} & \multicolumn{2}{|c|}{0,735} \\
\hline
\end{tabular}

a) Wohnort im Dezember des ersten Bezugsjahres

b) Wohnort im Dezember 1996

Tab. 3: Räumliche Verteilung von Sozialhilfe-Erstbeziehern jeweils im Dezember des ersten Bezugsjahres und im Dezember 1996

Spatial distribution of first-time social welfare recipients as of December of the first welfare support period and December 1996

Distribution spatiale des personnes ayant bénéficié pour la prermière fois de l'aide sociale, en décembre de la première année de perception et en décembre 1996

Quelle: eigene Berechnungen; Datenbais: Bielefelder Datenbank «Sozialhilfestatistik» (Sozialamt BielefELD; UNIVERSITÄT BIELEFELD) 


\begin{tabular}{|lllrr|}
\hline $\begin{array}{l}\text { Wohnort jeweils zum } \\
\text { Beginn des Sozialhilfebezugs } \\
\text { (Dezember 1992 bzw. 1993) }\end{array}$ & $\begin{array}{l}\text { Wohnort } \\
\text { Dezember 1996 }\end{array}$ & Mobilität & Prozent & Fälle \\
\hline übrige Stadt & übrige Stadt & kein Umzug & 61,6 & 375 \\
Armutsgebiet 1996 & Armutsgebiet 1996 & kein Umzug & 11,2 & 68 \\
übrige Stadt & übrige Stadt & Umzug & 20,7 & 126 \\
Armutsgebiet 1996 & übrige Stadt & Umzug & 1,3 & 8 \\
übrige Stadt & Armutsgebiet 1996 & Umzug & 3,1 & 19 \\
Armutsgebiet 1996 & Armutsgebiet 1996 & Umzug & 2,1 & 13 \\
\hline & & & $\mathbf{1 0 0 , 0}$ & $\mathbf{6 0 9}$ \\
\hline
\end{tabular}

Tab. 4: Mobilität und Richtung der Umzüge von Sozialhilfe-Erstbeziehern der Jahre 1992 und 1993

Mobility and direction of residential change of first-time social welfare recipients 1992 and 1993

Mobilité et direction des déménagements des personnes ayant bénéficié pour la première fois de l'aide sociale en 1992 ou 1993

Quelle: eigene Berechnungen; Datenbasis: Bielefelder Datenbank «Sozialhilfestatistik» (Sozialamt BiELEFELD; UNIVERSITÄT BIELEFELD)

formationen zu Bedarfsgemeinschaften zur Verfügung. Im Jahre 1996 umfaßte die SEDA 10.569 Fälle bzw. 23.549 Personen. Mit Hilfe des Aktenzeichens können die jahresweisen Querschnittsdaten zu einer Längsschnittdatei zusammengefügt werden.

Diese Längsschnittdaten erlauben, den Verlauf des Sozialhilfebezugs über einen längeren Zeitraum zu untersuchen. Aufgrund von Angaben zum Wohnort ist es zudem möglich, die räumliche Mobilität der Sozialhilfeempfänger nachzuverfolgen und so die oben aufgeführten Hypothesen zur Erklärung der überproportionalen Zunahme räumlicher Konzentration von Armut zu überprüfen.

In die Analysen werden im folgenden nur die acht Armutsgebiete einbezogen, deren überproportionaler Anstieg der Sozialhilfedichte im einzelnen überwiegend durch einen überdurchschnittlichen Zuwachs der Armutsbevölkerung begründet ist. Die Gruppe der Asylbewerber wird aus den Analysen ausgeschlossen, da diese mit dem Asylbewerberleistungsgesetz von 1993 nicht mehr sozialhilfeberechtigt sind und somit in der Sozialhilfestatistik nicht mehr ausgewiesen werden.

\subsection{Hypothese a) Zuzug der Armutsbevölkerung}

Als erstes wird untersucht, ob die überproportionale Zunahme der Armutsbevölkerung in den Armutsgebieten des Jahres 1996 durch einen verstärkten Zuzug von Sozialhilfeempfängern in diese Gebiete verursacht ist. Zu diesem Zweck werden Teilpopulationen sogenannter Erstbezugskohorten der Jahre 1992 bis 1995 genauer untersucht. Bei den einzelnen Kohorten handelt es sich um Bedarfsgemeinschaften von Deutschen (einschließlich Aussiedlern) und ansässigen
Ausländern, die in den jeweiligen Jahren erstmals Sozialhilfe bezogen haben und in mindestens fünf vorhergehenden Jahren nicht im Leistungsbezug standen. Für die Analyse ausgewählt wurden diejenigen Fälle der Kohorten, die bis zum Dezember 1996 kontinuierlich oder in mehreren Episoden Sozialhilfe bezogen haben. Innerhalb der Erstbezugskohorten erfüllen 256 von 1343 (1992), 353 von 1716 (1993), 616 von 2227 (1994) und 985 von 2218 (1995) Fällen dieses Kriterium. Da Analysen der Armutsverläufe einkommensarmer Bevölkerungsgruppen mit Hilfe des Sozio-ökonomischen Panels zeigen, daß Personen mit diskontinuierlichem Armutsverlauf in Phasen mit einem Einkommen oberhalb der Armutsgrenze in der Mehrzahl in einem armutsnahen Zustand verbleiben (Habich, Headey \& Krause 1991: 499), ist anzunehmen, daß auch die Wohnkarriere der Fälle mit mehreren Sozialhilfeepisoden weitgehend durch eine wirtschaftliche und häufig auch soziale Benachteiligung geprägt ist.

Um einen möglichen Zuzug von Sozialhilfeempfängern in die Armutsgebiete aufzuzeigen, wird die räumliche Verteilung der Teilpopulationen aus den Kohorten 1992 bis 1995 zu Beginn des Sozialhilfebezugs über die Gebietstypen «Armutsgebiete» und «übrige Stadt» der Verteilung im Dezember des Jahres 1996 gegenübergestellt. Läßt sich eine Veränderung der räumlichen Verteilung zugunsten der Armutsgebiete feststellen, so ist von einem verstärkten Zuzug von Sozialhilfeempfängern in diese Gebiete auszugehen.

Aus Tabelle 3 wird ersichtlich, da 3 sich die Anteile der Sozialhilfeempfänger in den Gebietstypen zu Beginn des Sozialhilfebezugs und am Ende des Beobachtungsfensters (Dezember 1996) entgegen der 


\begin{tabular}{|c|c|c|c|c|}
\hline \multirow[b]{2}{*}{ Merkmale } & & \multicolumn{2}{|c|}{ Wohnort 1996} & \multirow[b]{2}{*}{$\begin{array}{c}\text { insgesamt } \\
\text { (n) }\end{array}$} \\
\hline & & $\begin{array}{c}\text { übrige Stadt } \\
(\%)\end{array}$ & $\begin{array}{c}\text { Armuts- } \\
\text { gebiet (\%) }\end{array}$ & \\
\hline alle Bezieher & & 80,7 & 19,3 & 166 \\
\hline \multirow[t]{2}{*}{ Geschlecht } & männlich & 80,0 & 20,0 & 65 \\
\hline & weiblich & 81,2 & 18,8 & 101 \\
\hline \multirow[t]{3}{*}{ Alter } & unter 25 & 80,0 & 20,0 & 45 \\
\hline & $25-44$ & 84,0 & 16,0 & 94 \\
\hline & über 44 & 70,4 & 29,6 & 27 \\
\hline \multirow{6}{*}{ Haushalt } & Singles & 80,0 & 20,0 & 85 \\
\hline & Paare & 75,0 & 25,0 & 32 \\
\hline & alleinerziehende Eltern & 86,7 & 13,3 & 45 \\
\hline & sonstige Haushalte & 66,7 & 33,3 & 3 \\
\hline & HH ohne Kinder & 79,0 & 21,0 & 100 \\
\hline & $\mathrm{HH}$ mit Kindern & 83,1 & 16,9 & 65 \\
\hline \multirow[t]{2}{*}{ Nationalität } & Deutsche & 81,2 & 18,8 & 133 \\
\hline & ansässige Ausländer & 78,8 & 21,2 & 33 \\
\hline \multirow{7}{*}{ Einstiegsursachen } & Warten & 84,0 & 16,0 & 25 \\
\hline & Arbeitslosigkeit & 77,6 & 22,4 & 67 \\
\hline & unzureichendes Einkommen & n 92,3 & 7,7 & 13 \\
\hline & unzureichende Frührente & 100,0 & 0,0 & 5 \\
\hline & familiäre Ursachen & 80,0 & 20,0 & 25 \\
\hline & soziale Probleme & 70,0 & 30,0 & 10 \\
\hline & sonstige Ursachen & 81,0 & 19,0 & 21 \\
\hline gesundheitl. Probleme & Krankheit & 76,9 & 23,1 & 13 \\
\hline
\end{tabular}

Tab. 5: Verteilung von mobilen Sozialhilfe-Erstbeziehern der Jahre 1992 bzw. 1993 nach Gebietstyp und demographischen Merkmalen

Distribution of mobile first-time social welfare recipients 1992 and 1993 according to residential area and demographic characteristics

Distribution des personnes mobiles ayant bénéficié pour la première fois de l'aide sociale en 1992 ou 1993 par types de zone el caractéristiques démographiques

Datenbasis: Bielefelder Datenbank «Sozialhilfestatistik» (Sozialamt Bielefeld; Universität Bielefeld)

Ausgangshypothese kaum unterscheiden. Ein Prozeß der Segregation ist für die Erstbezugskohorten also nicht auszumachen. Der verstärkte Anstieg von Sozialhilfeempfängern in den Armutsgebieten des Jahres 1996 kann durch eine einseitige Zuwanderung von Sozialhilfeempfängern somit nicht erklärt werden.

Eine genauere Betrachtung der Wanderungsbewegungen zwischen den Gebietstypen zeigt, daß durchaus Zuzüge aus der «übrigen Stadt» in die Armutsgebiete stattgefunden haben (vgl. Tabelle 4). Diese wurden aber durch Fortzüge aus den Armutsgebieten so ausgeglichen, daß sich die räumliche Verteilung am Ende des Beobachtungszeitraumes nicht signifikant von der Ausgangsverteilung unterscheidet.

Zwar sind innerhalb der Gesamtgruppe kaum Segregationstendenzen zu beobachten, dennoch ist das Risiko des Zuzugs in ein Armutsgebiet für einzelne
Sozialhilfeempängergruppen durchaus verschieden. Tabelle 5 deutet - für die Erstbezugskohorten 1992 und 1993 zusammengefaßt - auf einen überproportionalen Zuzug einzelner Haushaltstypen in die Armutsgebiete hin. So sind Haushalte mit Personen im Alter von über 44 Jahren, Paare, sonstige Haushalte, Ausländer, Arbeitslose und Personen mit sozialen bzw. gesundheitlichen Problemen nach einem oder mehreren Umzügen in den Armutsgebieten überrepräsentiert.

Eine logistische Regression hat jedoch gezeigt, daß die Ungleichverteilungen zugunsten der Armutsgebiete für die genannten Haushaltsgruppen statistisch nicht signifikant sind. Ein signifikant höheres Risiko des Umzugs in ein Armutsgebiet liegt für die aufgeführten Haushaltstypen also nicht vor. Erstaunlich ist dieses Ergebnis insbesondere für die Gruppe der alleinerziehenden Eltern, da dieser Haushaltstyp mit erheblichen Zugangsproblemen auf dem Wohnungsmarkt 


\begin{tabular}{|lccccc|}
\hline Armutsgebiete 1996 & $\mathbf{1 9 9 2}$ & $\mathbf{1 9 9 3}$ & $\mathbf{1 9 9 4}$ & $\mathbf{1 9 9 5}$ & $\mathbf{1 9 9 6}$ \\
\hline Bauernschaft Schildesche & 1,1 & 2,2 & 6,5 & 5,3 & 9,2 \\
Milse & 0,4 & 0,8 & 2,5 & 3,4 & 3,0 \\
Osningpaß & 0,5 & 0,9 & 1,2 & 1,6 & 1,8 \\
Sieker & 1,1 & 1,5 & 1,7 & 1,4 & 1,5 \\
Pauluskirche & 1,3 & 2,2 & 2,2 & 2,1 & 1,4 \\
Baumheide & 0,9 & 1,5 & 2,0 & 1,4 & 1,4 \\
Stadtwerke & 0,7 & 1,8 & 1,5 & 2,3 & 1,2 \\
Oldentrup-West & 1,5 & 1,4 & 2,2 & 1,8 & 1,1 \\
\hline Bielefeld insgesamt & 0,7 & 0,9 & 1,1 & 1,2 & 1,1 \\
\hline
\end{tabular}

Tab. 6: Sozialhilfe-Erstbezieher pro Einwohner in den Armutsgebieten von 1996 und in der Gesamtstadt (in Prozent) Proportion of first-time social welfare recipients to total population in poverty areas and in the city 1996 (in percent) Nombre de personnes bénéficiant pour la première fois de l'aide sociale par habitant dans les zones de pauvreté de 1996 et dans l'ensemble de la ville (en pour-cent)

Quelle: eigene Berechnungen; Datenbasis: Stadt BIelefeld, Amt Für StadTforschung und Statistik; Bielefelder Datenbank «Sozialhilfestatistik» (Sozialamt Bielefeld; Universität Bielefeld)

zu rechnen hat (vgl. OSENBERG 1991) und damit tendenziell eher auf den Wohnungsbestand in unattraktiven städtischen Teilräumen und demzufolge auch in Armutsgebieten verwiesen ist.

\subsection{Hypothesen b) Verarmung und c) kommunale Zuweisungspraxis}

Als nächstes wird untersucht, ob der überdurchschnittliche Anstieg der Sozialhilfeempfänger in den Armutsgebieten durch eine verstärkte Verarmung der deutschen und seit längerem ansässigen ausländischen Bevölkerung bzw. durch eine überproportionale Zuweisung von Aussiedlern in die Armutsgebiete verursacht ist.

Um diese Hypothese zu überprüfen, wird für die einzelnen Armutsgebiete der Anteil der Erstbezieher eines Jahres an der Gesamtbevölkerung im Armutsgebiet berechnet. Liegt dieser Wert in einem Jahr über dem entsprechenden Wert für die Gesamtstadt, so kann von einer überdurchschnittlichen Verarmung bzw. von einer verstärkten Zuweisung von Aussiedlern in die Armutsgebiete ausgegangen werden.

Tabelle 6 verdeutlicht, daß in den untersuchten acht Armutsgebieten bis auf sehr wenige Ausnahmen überdurchschnittlich viele Personen neu Sozialhilfe beziehen. Insbesondere in den statistischen Bezirken Bauernschaft Schildesche und Milse (Milse erst seit 1995) ist der Anteil der Erstbezieher an der Bevölkerung sehr hoch.

Inwieweit die hohe Erstbezugsdichte in den Statistischen Bezirken auf eine Verarmung der ansässigen Bevölkerung oder auf eine Zuweisung von deutschen Aussiedlern zurückzuführen ist, kann aufgrund der
Tatsache, daß Aussiedler als solche im Datensatz nicht gesondert ausgewiesen sind, nicht unmittelbar beurteilt werden.

Einen Hinweis auf eine räumlich disparitäre Zuweisungspraxis der Kommune gibt jedoch die Lage von Übergangswohnheimen für Aussiedler im Stadtgebiet. Hier stellt sich heraus, daß die Armutsgebiete mit den höchsten Erstbezugsdichten (Bauernschaft Schildesche und Milse) allesamt auch Standorte von Übergangswohnheimen mit hoher Belegungszahl sind. Der Anteil von Aussiedlern in Übergangswohnheimen an der Gesamtbevölkerung ist hier besonderes hoch (vgl. Tabelle 7). Die hohe Erstbezugsdichte in diesen Gebieten ist also auch das Resultat einer verstärkten Zuweisung von Aussiedlern. In den übrigen Statistischen Bezirken ist die überdurchschnittliche Erstbezugsdichte vor allem durch eine verstärkte Verarmung der ansässigen Bevölkerung begründet.

Die aufgezeigte überdurchschnittliche Verarmung der Bevölkerung in den Armutsgebieten wird plausibel, wenn die sozio-demographische Bevölkerungsstruktur dieses Gebietstyps anhand von Daten der Volkszählung 1987 mit der in der «übrigen Stadt» verglichen wird. So ist aus Tabelle 8 zu entnehmen, daß Bevölkerungsgruppen mit erhöhtem Armutsrisiko (Ausländer, Ledige, Arbeiter, Personengruppen mit geringer formaler Bildung) in den acht Armutsgebieten mit überdurchschnittlicher Zunahme der Sozialhilfeempfänger gegenüber der «übrigen Stadt» wesentlich stärker vertreten sind. Die verstärkte Verarmung der Bevölkerung in bestimmten städtischen Teilgebieten und die daraus resultierende erhöhte Konzentration von Armut ist demnach als die Folge einer verschärften, dem Verarmungsprozeß zeit- 


\begin{tabular}{|llllll|}
\hline \multirow{2}{*}{ Statistischer Bezirk } & \multicolumn{5}{c}{ Aussiedler in Wohnheimen pro 100 Einwohner (Jahresende) } \\
\hline Bauernschaft Schildesche & $\mathbf{1 9 9 2}$ & $\mathbf{1 9 9 3}$ & $\mathbf{1 9 9 4}$ & $\mathbf{1 9 9 5}$ & $\mathbf{1 9 9 6}$ \\
Milse & 17,5 & 17,3 & 18,3 & 13,7 & 14,9 \\
Windelsbleiche & 10,4 & 8,3 & 7,6 & 6,3 & 6,0 \\
Windwehe & 6,1 & 4,9 & 4,9 & 4,7 & 5,9 \\
Kesselbrink & 7,7 & 6,4 & 6,2 & 7,1 & 4,8 \\
Königsbrügge & 7,2 & 3,4 & 3,5 & 3,6 & 4,5 \\
Brock & 2,6 & 2,3 & 2,3 & 2,0 & 2,4 \\
Eckardtsheim & 1,8 & 1,4 & 1,4 & 1,4 & 1,9 \\
Pappelkrug & - & 2,3 & 2,2 & 2,0 & 1,7 \\
Stieghorst & 2,6 & 1,9 & 1,9 & 1,7 & 1,1 \\
Sennestadt & 0,6 & 0,6 & 0,7 & 0,6 & 0,5 \\
Jöllenbeck-Ost & - & 0,5 & 0,5 & 0,5 & 0,5 \\
Fuhrpark & 0,5 & 0,3 & 0,3 & 0,1 & - \\
Bethel & 6,6 & - & - & - & - \\
Landgericht & 3,2 & 1,8 & 1,9 & 0,8 & - \\
Schildesche & 3,1 & 1,5 & 1,6 & 0,8 & - \\
Brands Busch & 4,1 & 1,8 & 1,9 & 0,7 & - \\
Dürkopp & 3,2 & - & - & - & - \\
Rosenhöhe & 2,5 & - & - & - & - \\
Südstadt & 1,2 & 0,8 & 0,8 & - & - \\
Frerks Hof & 0,6 & 0,7 & 0,7 & 0,9 & - \\
Vilsendorf & 0,9 & - & - & - & - \\
Hammer-Mühle & 0,4 & 0,1 & 0,1 & - & - \\
Sudbrack & - & - & - & - & - \\
\hline Bielefeld insgesamt & 0,1 & 0,1 & 0,1 & - & - \\
\hline
\end{tabular}

Tab. 7: Anzahl der Aussiedler in Übergangswohnheimen pro 100 Einwohner in den Statistischen Bezirken der Stadt Bielefeld 1992-1996 (jeweils am Jahresende)

Percentage of German immigrants in hostels per 100 inhabitants in the Bielefeld census districts 1992-1996 (yearend figures)

Nombre d'émigrants hébergés dans des foyers provisoires par centaine d'habitants dans les districts statistiques de la ville de Bielefeld, de 1992 à 1996 (données de la fin de chaque année)

Quelle: eigene Berechnungen; Datenbasis: Stadt Bielefeld, Amt Für Stadtforschung und Statistik; Stadt Bielefeld, Sozialamt

lich vorgelagerten Segregation sozialer Schichten und Risikogruppen zu werten.

\subsection{Hypothese d) Kumulation von Langzeitbeziehern}

Schließlich wird überprüft, ob die überdurchschnittliche Zunahme der Sozialhilfeempfänger durch eine Kumulation dauerhaft von Armut Betroffener in bestimmten Teilgebieten der Stadt verursacht ist (Hypothese d). Ausgangspunkt der Überlegung ist der Umstand, daß unter der Jahr für Jahr neu verarmenden Bevölkerung Teilgruppen eine lange oder sehr lange Dauer des Sozialhilfebezugs aufweisen. Unter der Voraussetzung einer annähernd gleichen Struktur des Sozialhilfebezugs für die nachfolgenden Jahre kommt es zu einer Kumulation der dauerhaft von Armut betroffenen Bevölkerung und damit zu einem
Anstieg der Armutsbevölkerung insgesamt. Räumlich differenziert ist ein überdurchschnittlicher Anstieg der Armutsbevölkerung aufgrund der beschriebenen Kumulationsprozesse in den Teilgebieten der Stadt zu erwarten, in denen der Anteil dauerhaft von Armut Betroffener an der Erstbezugskohorte für jedes Jahr über dem Durchschnitt der Gesamtstadt liegt.

Um nun festzustellen, inwieweit der überdurchschnittliche Anstieg der Sozialhilfeempfänger in den Armutsgebieten von 1996 allein durch die Kumulation eines erhöhten Anteils an Langzeitbeziehern verursacht ist, wird der Effekt der Kumulation sowohl für die Gesamtstadt als auch für die Armutsgebiete von 1996 über einen festgelegten Zeitraum simuliert. Am Ende des Beobachtungszeitraums kann für jeden Gebiets- 


\begin{tabular}{|lcc|}
\hline Ausprägung & $\begin{array}{c}\text { Armutsgebiete } \\
(\%)\end{array}$ & $\begin{array}{c}\text { ibrige Stadt } \\
\text { (\%) }\end{array}$ \\
\hline Bevölkerung & 19,8 & 16,6 \\
Personen unter 18 Jahren & 14,0 & 8,5 \\
Ausländer & 40,8 & 38,2 \\
Ledige & 45,5 & 47,8 \\
Verheiratete & 8,8 & 9,9 \\
Verwitwete & 5,2 & 3,5 \\
Erwerbslose & 28,5 & 28,2 \\
Arbeitslose, Rentner, Pensionäre & & 37,7 \\
Haushalte & 41,0 & 4,8 \\
HH mit 1 Person & 6,0 & 38,8 \\
HH mit 5 und mehr Personen & & 43,9 \\
Erwerbstätige & 50,8 & 8,6 \\
Arbeiter & 37,2 & 7,8 \\
Angestellte & 5,7 & \\
Beamte, Richter, Soldaten & 5,6 & 57,9 \\
Selbständige & & 23,8 \\
Schulabschluß & 66,1 & 9,3 \\
Hauptschule & 20,0 & 18,3 \\
Realschule & 7,9 & 9,5 \\
berufsbildender Abschluß & 13,9 & \\
Hochschulreife, Fachhochschulreife & 5,8 & \\
Fachhochschule, Universität & & \\
\hline
\end{tabular}

Tab. 8: Sozio-demographische Charakteristika der Bevölkerung in den untersuchten Armutsgebieten und in der «übrigen Stadt»

Socio-demographic characteristics of the population in the investigated poverty areas and in other areas of the city

Caracteréristiques socio-démographiques de la population dans les zones de pauvreté étudiées et dans le reste de la ville

Quelle: eigene Berechnungen; Datenbasis: Volkszählung 1987; STADT Bielefeld, Amt Für STADTFORSCHUng UND STATISTIK

typ bestimmt werden, um welchen Faktor, ausgehend von der Anzahl der Empfänger der Ausgangskohorte, die Zahl der Sozialhilfeempfänger allein aufgrund von Kumulationsprozessen zugenommen hat. Ist der Zunahmefaktor für die Armutsgebiete größer als für die Gesamtstadt, so ist anzunehmen, daß der überdurchschnittliche Anstieg von Sozialhilfeempfängern in den Armutsgebieten auch durch Prozesse der Kumulation eines höheren Anteils von Dauerempfängern verursacht wurde.

Bevor im einzelnen auf die Zunahmefaktoren der Gesamtstadt und der Armutsgebiete eingegangen wird, soll das Verfahren der Simulation und die Ableitung des Zunahmefaktors kurz an einem Beispiel verdeutlicht werden.

Eine sogenannte Überlebenskurve (Abbildung 2) zeigt zunächst für zwei Gebietstypen $\mathrm{A}$ und $\mathrm{B}$ den Anteil an Sozialhilfeempfängern, der nach einer bestimmten Zeit noch immer im Bezug ist. Deutlich wird, daß der Anteil von Sozialhilfeempfängern mit langer Bezugsdauer (länger als ein Jahr) in Gebietstyp B zu allen Zeitpunkten deutlich höher ausfällt als in Gebietstyp A. Im Gebietstyp B ist also aufgrund von Kumulationsprozessen eines höheren Anteils von Langzeitbeziehern mit einem gegenüber Gebietstyp A vergleichsweise stärkerem Zuwachs von Sozialhilfeempfängern zu rechnen.

Abbildung 3 verdeutlicht den Proze $\beta$ der Kumulation von Langzeitempfängern für die Gebietstypen A und B über einen Zeitraum von sechs Jahren. Die Struktur der Dauer des Sozialhilfebezugs bleibt in den jährlich aufeinander folgenden Bezugskohorten I-VI konstant. Betrachtet man für beide Gebietstypen zunächst die erste Kohorte (I), so scheidet (per definitionem) spätestens nach 24 Monaten der letzte kurzfristige 


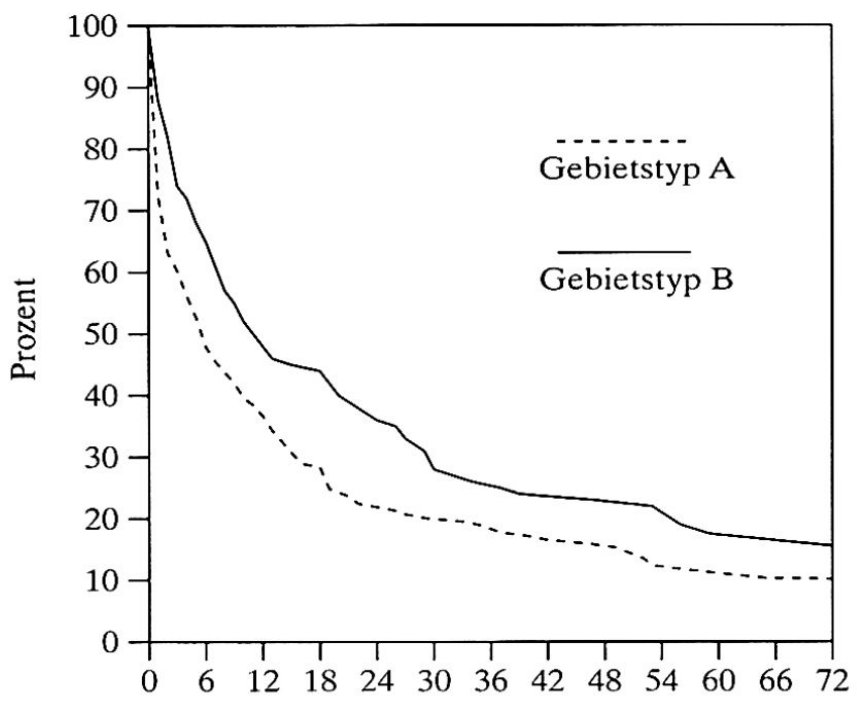

Dauer des Sozialhilfebezugs von Beginn des Bezugs in Monaten

Abb. 2: Dauer des Sozialhilfebezugs in zwei Gebietstypen (Beispiel)

Duration of social welfare benefit by type of residential area (example)

Durée de la période de perception de l'aide sociale dans deux types de zone (exemple)

Quelle: eigene Berechnungen; fiktive Daten
Sozialhilfeempfänger (Bezugszeit bis zu einem Jahr) aus der Sozialhilfe aus. Die langfristigen Empfänger der ersten Kohorte (Bezugszeit über einem Jahr) beziehen weiterhin Sozialhilfe und kumulieren mit dem Eintreten weiterer Kohorten (II-VI) über einen bestimmten Zeitraum hinweg auf. Für beide Gebietstypen gilt:Während sich der Anteil von Kurzzeitbeziehern aufgrund des sukzessiven Ausstiegs dieser Gruppen nach einem Jahr stabilisiert, nimmt der Anteil der Haushalte mit langer Verweildauer beständig zu.

Von Bedeutung ist, daß die Zahl der Sozialhilfeempfänger aufgrund des erhöhten Anteils von Langzeitbeziehern im Gebietstyp B wesentlich stärker ansteigt als im Gebietstyp A. Am Ende des Betrachtungszeitraums hat sich die Zahl der Sozialhilfeempfänger im Gebietstyp B um einen Faktor von 194 Prozent gegenüber dem ursprünglich in der Ausgangskohorte I vorliegenden Bestand an Sozialhilfeempfängern erhöht, während der Anstieg in Gebietstyp A nur um einen Faktor von 132 Prozent erfolgt ist. Die Zahl der in der Ausgangskohorte vertretenen Sozialhilfeempfänger beträgt nach 12 Monaten (nachdem alle Fälle dieser Kohorte in den Bezug von Sozialhilfe eingetreten sind) «nur» ca. 50 Prozent (nicht etwa 100 Prozent), da ca. 50 Prozent der Empfänger dieser ersten Kohorte I (vgl. auch Abbildung 2) am Ende des Jahres (nach 12 Monaten) bereits wieder aus dem Bezug ausgestiegen sind. Aufgrund des beständig höheren Anteils von Langzeitbeziehern in Gebietstyp
Gebietstyp A (geringer Anteil an Langzeitbeziehern)

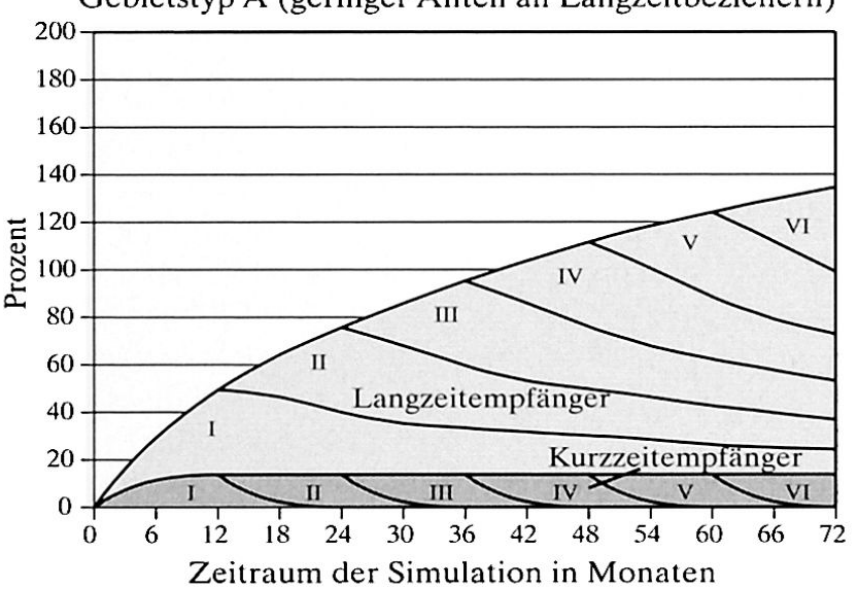

Gebietstyp B (hoher Anteil an Langzeitbeziehern)

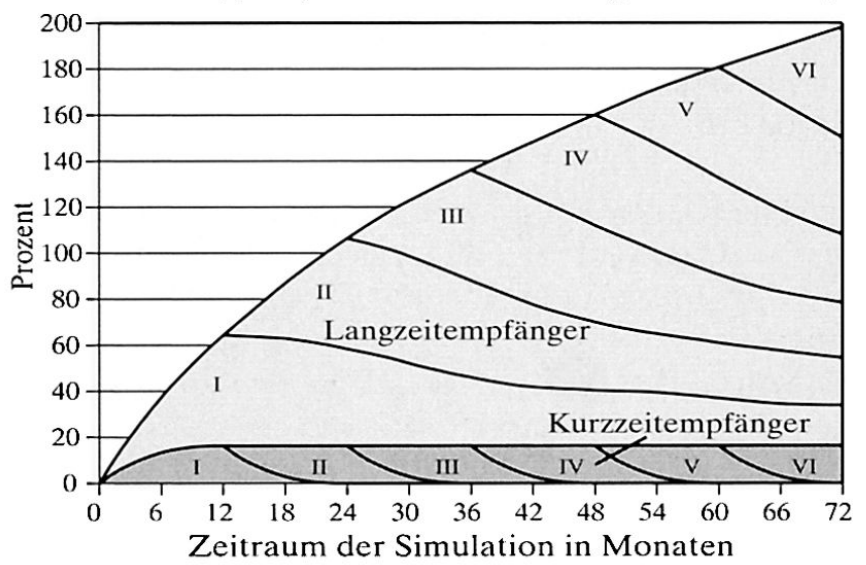

Abb. 3: Simulation einer jahresweisen Entwicklung der Zahl der Sozialhilfeempfänger über einen Zeitraum von sechs Jahren nach Art der Empfänger für Gebietstyp A und B (Beispiel)

Simulation of development of the numer of social welfare recipients over a period of six years by type of recipient in area type $A$ and $B$ (example)

Simulation d'une évolution annuelle du nombre de bénéficiaires de l'aide sociale sur une période de six ans par types de bénéficiaires dans les types de zone A et B (exemple)

Quelle: eigene Berechnungen; fiktive Daten 


\begin{tabular}{|lc|}
\hline Kohorte 1992 & Zunahmefaktor \\
\hline Gesamtstadt & 242,3 \\
Armutsgebiete 1996 insgesamt & 236,3 \\
Bauernschaft Schildesche & $(167,2)$ \\
Baumheide & 253,6 \\
Sieker & 221,7 \\
Pauluskirche & 200,2 \\
Stadtwerke & $(250,6)$ \\
Osningpaß & $(256,3)$ \\
Milse & $(213,9)$ \\
Oldentrup-West & 309,9 \\
\hline
\end{tabular}

Anmerkung: Werte in Klammern beziehen sich auf eine Fallzahl von unter 20 und können daher allenfalls als Trend interpretiert werden.

Tab. 9: Zunahmefaktor der Anzahl von Sozialhilfeempfängern nach einer Simulation der Kumulation von Langzeitbeziehern (Bezug von einem Jahr und länger) über einen Zeitraum von sechs Jahren

Factor by which social welfare recipients are expected to increase according to a simulation of an accumulation process of long-term (over a year) social assistance recipients over a period of six years

Facteur de progression du nombre de bénéficiaires de l'aide sociale d'après une simulation de l'accumulation des bénéficiaires à long terme (perception durant une année ou plus) sur une période de six ans

Quelle: eigene Berechnungen; Datenbasis: Bielefelder Datenbank «Sozialhilfestatistik» (Sozialamt BieLEFELD; UNIVERSITÄT BIELEFELD)

B hat sich also die Zahl der Sozialhilfeempfänger durch Prozesse der Kumulation in deutlich stärkerem Maße erhöht.

In Tabelle 9 sind die Zunahmefaktoren für die Gesamtstadt und die Armutsgebiete von 1996, die durch eine Simulation von Kumulationsprozessen über einen Zeitraum von sechs Jahren ermittelt wurden, aufgeführt. Es zeigt sich, daß der Zunahmefaktor für die Armutsgebiete insgesamt mit einem Wert von 236 Prozent geringer ausfällt als in der Gesamtstadt mit 242 Prozent. Dieses Ergebnis ist auf einen leicht höheren Anteil von Langzeitempfängern in der Gesamtstadt gegenüber den Armutsgebieten zurückzuführen (vgl. Abbildung 4). Ein überdurchschnittlicher Anstieg der Sozialhilfeempfänger ist also durch Effekte der Kumulation von Langzeitbeziehern für die Armutsgebiete insgesamt nicht zu erklären. Im Gegenteil ist ein solcher Effekt nach den Ergebnissen eher in der Gesamtstadt zu erwarten.

In einzelnen Armutsgebieten (Baumheide, OldentrupWest) liegt der Zunahmefaktor jedoch über dem
Wert in der Gesamtstadt, so daß der überdurchschnittliche Anstieg der Sozialhilfeempfänger in diesen Armutsgebieten durchaus auf Effekte der Kumulation von Langzeitempfängern zurückgeführt werden kann. Über den Aspekt eines kumulativen Anstiegs der Armutsbevölkerung hinaus deuten die überproportionalen Anteile von Langzeitempfängern in einzelnen Armutsgebieten auf die besondere soziale Problematik in diesen Gebieten hin. Aufgrund der räumlichen Konzentration eines langfristigen Sozialhilfebezugs steigt in einzelnen Wohnquartieren die Gefahr der Herausbildung von destruktiven Lebensverhältnissen und Milieus (siehe oben) erheblich an.

\section{Fazit}

Mit der gesamtstädtischen Zunahme der Zahl von Sozialhilfeempfängern in den 1990er Jahren ist das Ausmaß der räumlichen Konzentration von Armut

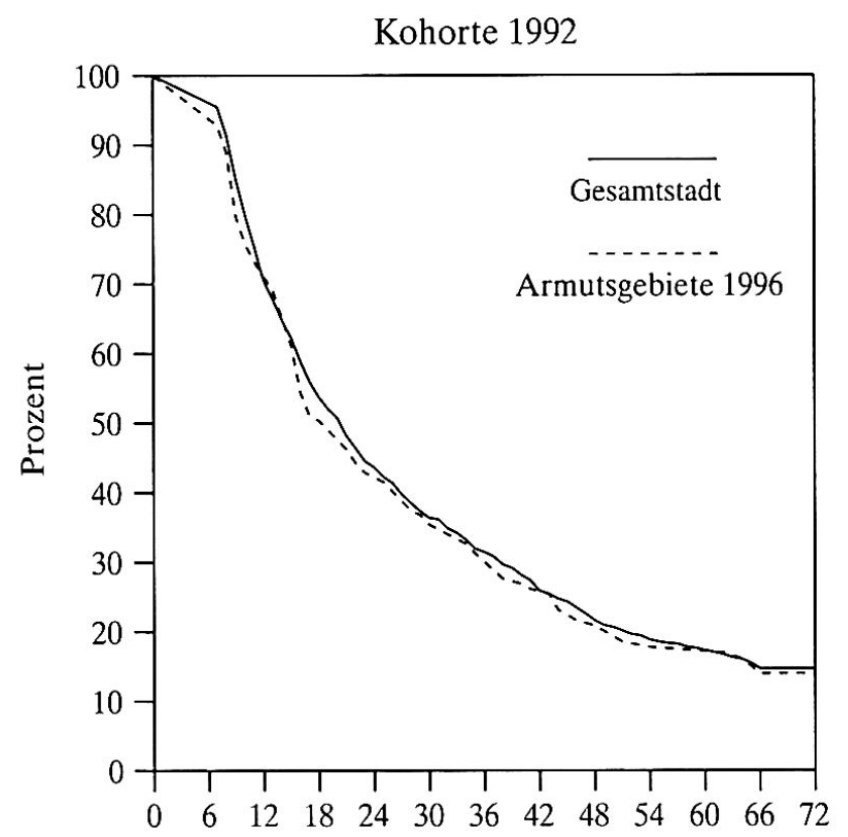

Dauer der Armutslage von Beginn des Bezugs in Monaten

Abb. 4: Dauer des Sozialhilfebezugs in der Gesamtstadt und in den Armutsgebieten 1996 (Zeiten des Nichtbezugs sind in der Gesamtdauer enthalten)

Duration of social welfare benefits in the city on the whole and in poverty areas 1996 (periods without financial assistance are included in the total duration)

Durée de la période de perception de l'aide sociale dans l'ensemble de la ville et dans les zones de pauvreté de 1996 (les périodes de non perception sont inclues dans la durée totale)

Quelle: eigene Berechnungen; Datenbasis: Bielefelder Datenbank «Sozialhilfestatistik» (Sozialamt Biflefeld; UNIVERSTTÄT BIELEFELD) 
- gemessen durch die Sozialhilfedichte - in vielen der bereits zu Beginn der 1990er Jahre bestehenden Armutsgebiete in Bielefeld deutlich angestiegen. Zusätzlich sind aber auch neue Gebiete hoher Sozialhilfedichte entstanden. Eine Zerlegung des Gesamteffekts eines überdurchschnittlichen Anstiegs der Sozialhilfedichte in den Armutsgebieten mit Hilfe eines vom Autor entwickelten, der Shift-Analyse ähnlichen Verfahrens hat gezeigt, daß dieser überwiegend durch eine verstärkte Zunahme der Zahl von Sozialhilfeempfängern verursacht ist.

Längsschnittanalysen der räumlichen Verteilung von Sozialhilfe-Erstbezugskohorten der Jahre 1992 bis 1995 für Bielefeld verdeutlichen, daß der überproportionale Anstieg der Sozialhilfeempfänger in den Armutsgebieten nicht durch einen einseitig gerichteten Zuzug bereits im Sozialhilfebezug stehender Bevölkerungsgruppen in die Armutsgebiete erklärt werden kann. Zwar ist das Risiko des Zuzugs in ein Armutsgebiet für einzelne Sozialhilfeempfängergruppen überproportional hoch, insgesamt fand aber innerhalb der Erstbezugskohorten keine statistisch signifikante Umverteilung zugunsten der Armutsgebiete statt. Vielmehr - so zeigen die Analysen - ist der starke Anstieg der Zahl der Sozialhilfeempfänger durch eine erhöhte Verarmung der Bevölkerung in den Armutsgebieten und eine kommunale Zuweisung von Aussiedlern in die betreffenden Gebiete begründet. Bei einigen Armutsgebieten ist davon auszugehen, daß der überproportionale Anstieg der Sozialhilfeempfänger auch durch eine Kumulation von langfristigen Sozialhilfebeziehern verursacht ist.

Die Analysen zeigen somit, daß - auch in Zeiten eines angespannten Vermietungsmarktes im Teilsegment preisgünstiger Wohnungen - nicht per se von einer durch Zuzug bedingten räumlichen Polarisierung von Sozialhilfeempfängern auszugehen ist. Zum einen haben die Analysen der Mobilität und Richtung von Umzügen gezeigt, daß ein großer Teil der untersuchten Sozialhilfeempfänger innerhalb des Beobachtungsfensters von immerhin vier Jahren gar nicht umgezogen ist und somit an dem Wohnort verbleibt, an dem der Bezug von Sozialhilfe begonnen hat. Dies könnte durchaus als eine «erzwungene Seßhaftigkeit» (KrEIBICH 1979: 156) interpretiert werden. Zum anderen verdeutlicht die hohe Zahl der Umzüge innerhalb der «übrigen Stadt» oder auch - ausgehend von Armutsgebieten - in die «übrige Stadt», daß auch außerhalb der Armutsgebiete für Sozialhilfeempfänger durchaus «Nischen» preisgünstiger und damit für diese Gruppen zugänglicher Wohnungen existieren.

Wenn auch ein einseitiger, auf die Armutsgebiete gerichteter Zuzug von Sozialhilfeempfängern nicht nachzuweisen ist, so deutet jedoch der Tatbestand einer verstärkten Verarmung der Bevölkerung in den
Armutsgebieten auf eine sich über einen längeren Zeitraum vollziehende, breitere Segregation sozial schwacher Bevölkerungsgruppen mit erhöhtem Verarmungsrisiko hin. Eine Strategie der Vermeidung zunehmender räumlicher Konzentrationen von Armut müßte demnach zukünftigen Prozessen der residentialen Segregation von sozial schwachen Bevölkerungsgruppen entgegenwirken, darüber hinaus aber auch eine langfristige Entflechtung bereits bestehender sozialräumlicher Strukturen einleiten. Sie muß bereits bei den von Armut bedrohten Haushalten ansetzen, um den der Verarmung vorgelagerten Segregationsprozessen Rechnung zu tragen.

Da die sozialräumliche Separierung der städtischen Bevölkerung im wesentlichen durch Mechanismen des Wohnungsmarktes vermittelt ist, muß eine solche Strategie einerseits eine Politik der Bestandssicherung des preiswerten Wohnraums in sozial gemischten Gebieten beinhalten (vgl. Distler 1990), andererseits Elemente einer aktiven, sozialräumlich ausgeglichenen Wohnungsbaupolitik nach dem Prinzip der Standortgerechtigkeit (vgl. KREIBICH 1990) einschließen. Im Hinblick auf die Standortplanung von Übergangswohnheimen für Aussiedler sind die Kommunen gefordert, trotz der $\mathrm{zu}$ erwartenden Widerstände von seiten der Bevölkerung in den betroffenen Stadtgebieten, eine Politik der sozial gerechten Standorte durchzusetzen.

\section{Literatur}

Andress, H.J. \& P. Kemper (1992): Möglichkeiten der Analyse prozeßproduzierter Sozialhilfedaten anhand eines Beispiels der AKD-KDN-Abrechnungsdaten in NRW. - In: JOHRENDT, N. \& H.R. SCHNEIDER (Hrsg.): Computergestützte Sozialberichterstattung und Sozialplanung, Bielefeld: 88-108.

BAHRDT, H.P. (1974): Die Zusammensetzung der Quartiersbevölkerung. - In: Herlyn, U. (Hrsg.): Stadt und Sozialstruktur, München: 172-186.

Blasius, J. \& J. Dangschat (Hrsg.) (1990): Gentrification. - Frankfurt am Main u.a.: Campus.

DistLeR, G. (1990): Der Verlust preisgünstigen Wohnraums - kann er abgeschwächt, können seine Folgen gemildert werden? Das Beispiel München. - In: BLAsius, J. \& J. Dangschat (Hrsg.): Gentrification, Frankfurt am Main, u.a.: 114-128.

FARWICK, A. (1998): Soziale Ausgrenzung in der Stadt. Struktur und Verlauf von Sozialhilfebedürftigkeit in städtischen Armutsgebieten. - In: Geographische Rundschau 50: 146-153.

FARWICK, A. (2001): Segregierte Armut. Ursachen und soziale Folgen der räumlichen Konzentration von Sozialhilfeempfängern am Beispiel der Städte Bremen und Bielefeld. - Opladen: Leske + Budrich.

Freie und Hansestadt Hamburg (1993): Armut in 
Hamburg. - = Beiträge zur Sozialberichterstattung, Hamburg: Eigenverlag.

Friedrichs, J. \& R. KeCSKes (Hrsg.) (1996): Gentrification. Theorie und Forschungsergebnisse. - Opladen: Westdeutscher Verlag.

Froessler, R. (1994): Integrierende Politik. Aufgaben, Inhalte und Formen staatlicher Programme zur Erneuerung benachteiligter Quartiere. - In:FrOESSLER, R. et al. (Hrsg.): Lokale Partnerschaften, Basel u.a.: 8-35.

Habich, R., Headey, B. \& P. Krause (1991): Armut im Reichtum. Ist die Bundesrepublik Deutschland eine Zwei-Drittel-Gesellschaft? - In: RendTEl, U. \& G. WAGNER (Hrsg.): Lebenslagen im Wandel, Frankfurt am Main u.a.: 488-509.

HÄUSSERMANN, H. \& W. Siebel (1987): Neue Urbanität. - Frankfurt am Main: Suhrkamp.

Häussermann, H. \& W. Siebel (1996): Soziologie des Wohnens. - Weinheim: Juventa.

Häussermann, H. \& W. Siebel (2000): Wohnverhältnisse und Ungleichheit. - In: HARTH, A., SCHELLER, G. \& W. Tessin (Hrsg.): Stadt und soziale Ungleichheit, Opladen: $120-140$.

Herlyn, U. (1974): Soziale Segregation. - In: Pehnt, W. (Hrsg.): Die Stadt in der Bundesrepublik Deutschland, Stuttgart: 89-106.

Hess, H. \& A. Mechlen (1973): Ghetto ohne Mauern. Ein Bericht aus der Unterschicht. - Frankfurt am Main: Suhrkamp.

KlaGge, B. (1998): Armut in westdeutschen Städten. Ursachen und Hintergründe für die Disparitäten städtischer Armut. - In: Geographische Rundschau 50: 139-145.

Kreibich, V. (1979): Zum Zwangscharakter räumlicher Mobilität. - In: JÜNGST, P. \& H.-J. Schulze-Göbel (Hrsg.): Stadt und Gesellschaft. Sozioökonomische Aspekte von Stadtentwicklung. - = Urbs et Regio 13 (Sonderband), Kassel: 153-210.

KREIBICH, V. (1985): Wohnungsversorgung und Wohnstandortverhalten. - In: Friedrichs, J. (Hrsg.): Die Städte in den achtziger Jahren, Opladen: 181-195.

Kreibich, V. (1990): Die Gefährdung preisgünstigen Wohnraums durch wohnungspolitische Rahmenbedingungen. - In: Blasius, J. \& J. Dangschat (Hrsg.): Gentrification, Frankfurt am Main u.a.: 51-68.

Kronauer, M. (1997): «Soziale Ausgrenzung» und «Underclass». Über neue Formen der gesellschaftlichen Spaltung. - In: Leviathan 25: 28-49.

LANDESHAuPTSTADT STUTTGART (1989): Räumliche Strukturen der Armut. - = Statistischer Informationsdienst, Beiträge aus Statistik und Stadtforschung, Sonderheft 12/1989, Statistisches Amt der Landeshauptstadt Stuttgart: Eigenverlag.

Mollenkopf, J.H. \& M. Castells (1991): Dual City: Restructuring New York. - New York: Russell Sage Foundation.

NAROSKa, H.-J. (1987): Sozialer Wohnungsbau als lokale Manövriermasse. - In: Neue Praxis 17: 489-505. OSENBERG, H. (1991): Die Versorgungsfunktion eines belegungsgebundenen Wohnungsbestands auf großstädtischen Wohnungsmärkten. Umzugsbefragung in Dortmund, Köln und München. - In: Informationen zur Raumentwicklung: 315-328.

ReICHERTZ, S. (1987): Verarmung durch Arbeitslosigkeit - dargestellt am Beispiel Essen. - In: Informationen zur Raumentwicklung: 551-566.

SCHÄTZL, L. (1981): Wirtschaftsgeographie 2. Empirie. - Paderborn u.a.: UTB Schöningh.

VAsKovics, L.A. (1976): Segregierte Armut. Randgruppenbildung in Notunterkünften. - Frankfurt am Main u.a.: Campus

VASKovics, L.A. (1982): Residentiale Segregation und soziale Probleme. - In: VAsKovics, L.A. (Hrsg.): Raumbezogenheit sozialer Probleme, Opladen: 200-227.

\section{Zusammenfassung: Armut in der Stadt - Prozesse der Ausweitung und Verfestigung räumlich segregierter Armut am Beispiel der Stadt Bielefeld}

Mit dem Anstieg der Armut in den Städten (West-) Deutschlands hat sich auch das Ausmaß segregierter Armut innerhalb der Städte deutlich verschärft. Analysen der Daten von Sozialhilfe-Erstbezugskohorten zeigen, daß der starke Anstieg der Sozialhilfedichte in bestimmten städtischen Teilgebieten insbesondere durch eine überdurchschnittliche Verarmung der Bevölkerung und darüber hinaus durch eine kommunale Zuweisung von Aussiedlern in die betroffenen Gebiete verursacht ist. Ein einseitig auf die Armutsgebiete gerichteter Zuzug von Sozialhilfeempfängern konnte dagegen nicht festgestellt werden.

\section{Summary: Urban Poverty - Processes leading to the strengthening and expansion of spatially segregated poverty. Case study Bielefeld.}

With growing poverty in (West-)German cities, segregated poverty within these cities has increased noticeably as well. Analyses of data related to social welfare recipient cohorts indicate that the increasing spatial segregation of poverty has mostly been caused by the impoverishment of those already living within these areas and additionally by an allocation of ethnic German immigrants from Eastern Europe to these areas by the local government. A strong inward movement of social welfare recipients into the poverty areas could not be identified.

Résumé: La pauvreté en ville - processus d'extension et de durcissement de la pauvreté ségrégative au niveau spatial, l'exemple de la ville de Bielefeld Avec l'augmentation de la pauvreté dans les villes d'Allemagne (de l'Ouest), on assiste aussi à un net accroissement de la pauvreté ségrégative à l'intérieur de ces villes. En analysant les données des cohortes de 
bénéficiaires de l'aide sociale, on s'aperçoit que la forte densification de l'aide sociale dans certains quartiers est surtout due à une paupérisation de leurs habitants supérieure à la moyenne, à laquelle vient s'ajouter l'établissement par les autorités locales d'émigrants (d'Europe de l'Est) dans les mêmes zones. Par contre, aucun afflux de bénéficiaires de l'aide sociale dirigé uniquement sur ces zones de pauvreté n'a pu être observé.

Dr. Andreas Farwick, Institut für Geographie (FB 8), Universität Bremen, Postfach 330440, D-28334 Bremen.

e-mail: afarwick@uni-bremen.de

Manuskripteingang/received/rentrée de manuscrit: 23.1.2001

Annahme zum Druck/Accepted for publication/ acceptation à l'impression: 21.6.2001 